\title{
Impact of Near-Surface Thermal Stresses on Interfacial Reliability of Through-Silicon Vias for 3-D Interconnects
}

\author{
Suk-Kyu Ryu, Kuan-Hsun Lu, Xuefeng Zhang, Jang-Hi Im, Senior Member, IEEE, \\ Paul S. Ho, Fellow, IEEE, and Rui Huang
}

\begin{abstract}
Continual scaling of on-chip wiring structures has brought significant challenges for materials and processes beyond the 32-nm technology node in microelectronics. Recently, 3-D integration with through-silicon vias (TSVs) has emerged as an effective solution to meet the future interconnect requirement. Among others, thermomechanical reliability is a key concern for the development of TSV structures used in die stacking as 3-D interconnects. This paper examines the effects of thermally induced stresses on the interfacial reliability of TSV structures. First, 3-D distribution of the thermal stress near the TSV and the wafer surface is analyzed. Using a linear superposition method, a semianalytic solution is developed for a simplified structure consisting of a single TSV embedded in a silicon ( $\mathrm{Si}$ ) wafer. The solution is verified for relatively thick wafers by comparing to numerical results from finite element analysis (FEA). The stress analysis suggests interfacial delamination as a potential failure mechanism for the TSV structure. An analytical solution is then obtained for the steady-state energy release rate as the upper bound for the interfacial fracture driving force, while the effect of crack length is evaluated numerically by FEA. With these results, the effects of the TSV dimensions (e.g., via diameter and wafer thickness) on the interfacial reliability are elucidated. Furthermore, the effects of via material properties and dielectric buffer layers are discussed.
\end{abstract}

Index Terms-Interfacial delamination, thermal stress, through-silicon via (TSV), 3-D interconnects.

\section{INTRODUCTION}

C ONTINUAL scaling of microelectronic devices has brought serious challenges to the materials and processes of on-chip interconnects beyond the 32-nm technology node [1]. The 3-D integration presents an effective solution as a system approach, which has generated significant interests recently to develop 3-D interconnects [2]-[4]. A critical structural element in 3-D integration is the through-silicon via (TSV), which directly connects stacked structures die to die. The TSVs may assume various structural configurations such as fully

Manuscript received June 24, 2010; accepted August 9, 2010. Date of publication August 23, 2010; date of current version March 9, 2011. This work was supported in part by Semiconductor Research Corporation.

S.-K. Ryu and R. Huang are with the Department of Aerospace Engineering and Engineering Mechanics, University of Texas, Austin, TX 78712 USA (e-mail: ruihuang@mail.utexas.edu).

K.-H. Lu, J.-H Im, and P. S. Ho are with the Microelectronics Research Center, University of Texas, Austin, TX 78712 USA (e-mail: paulho@mail. utexas.edu).

X. Zhang is with Advanced Micro Devices, Austin, TX 78735 USA (e-mail: Frank.Zhang@amd.com).

Color versions of one or more of the figures in this paper are available online at http://ieeexplore.ieee.org.

Digital Object Identifier 10.1109/TDMR.2010.2068572

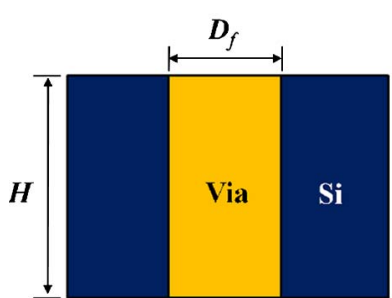

(a)

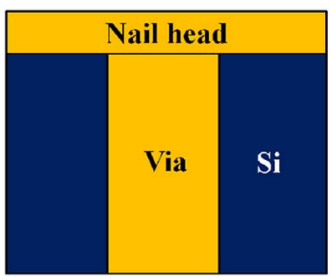

(c)

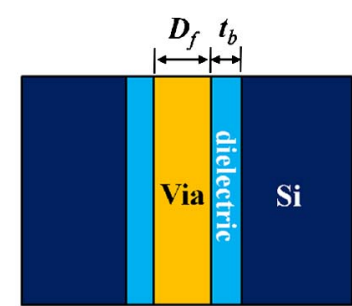

(b)

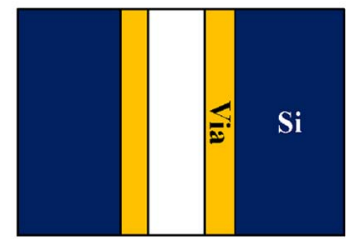

(d)
Fig. 1. Schematics of TSVs in various structural forms. (a) Fully filled TSV. (b) TSV with a dielectric buffer. (c) TSV with a nail head. (d) Annular TSV.

filled TSV, annular TSV, TSV with "nail head," and TSV with dielectric buffer layers (see Fig. 1). Use of TSVs in 3-D integration can effectively improve system performance and reduce manufacturing costs [3]-[7].

Due to mismatch in the coefficients of thermal expansion (CTEs) of the via materials and $\mathrm{Si}$, thermal stresses are ubiquitously induced during processing and thermal cycling of TSV structures, which can potentially degrade the performance of stress-sensitive devices around the TSVs [8], [9] or drive crack growth in 3-D interconnects [9]-[13]. Therefore, the success of 3-D integration largely relies on the characteristics of thermomechanical stresses developed in the system and its impact on reliability. Finite element methods have been used to numerically analyze the thermomechanical stresses in 3-D integrated structures [9]-[13], typically complicated by specific material processes and structural designs. To assess the thermomechanical reliability of TSV structures, the driving forces for both cohesive and interfacial crack growths were calculated based on fracture mechanics [12], [13]. In addition to these numerical studies, a simple analytical approach based on a 2-D model was used to analyze the thermomechanical interactions in TSV arrays [14]. However, the 2-D solution does not capture the 3-D nature of the stress field near the wafer surface around a TSV. Determination of the 3-D near-surface stress distribution is critical due to the fact that the active devices are usually located near the wafer surface. To date, a systematic understanding of the near-surface thermal stress 


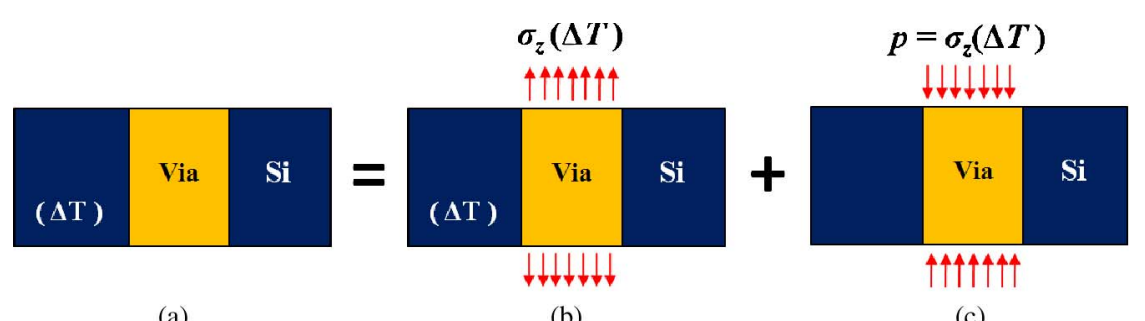

(a)

(b)

(c)

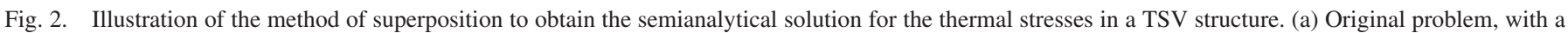
thermal load and traction-free surfaces. (b) Problem A, with a thermal load and surface traction. (c) Problem B, with surface load only.

distribution and its impact on thermomechanical reliability of TSVs has not been established.

In this paper, a semianalytic 3-D solution is developed for an isolated TSV embedded in the silicon wafer [Fig. 1(a)], which compares closely with numerical results obtained by finite element analysis (FEA) for TSV structures with relatively thick wafers. We then focus on the interfacial reliability of TSV, for which an analytical solution is obtained for the steady-state energy release rate as the upper bound for the fracture driving force. Based on these results, the effects of the TSV materials and geometries on interfacial reliability are investigated.

\section{ThreE-Dimensional Stress AnAlysis}

\section{A. Semianalytical Approach}

Consider a single TSV embedded in an infinite Si wafer [Fig. 2(a)]. The stress field induced by differential thermal expansion in the via and $\mathrm{Si}$ is 3-D in nature. As a prerequisite for the study of stress-related phenomena, we assume in this paper that all materials are isotropic and linearly elastic. Under the assumption of linear elasticity, the stress field in the TSV structure can be obtained by superposition of the two problems shown in Fig. 2. In Problem A [Fig. 2(b)], the system is subjected to a thermal loading $(\Delta T)$ and a uniform stress $\left(\sigma_{z}\right)$ on the surfaces of the via, so that the stress field is homogeneous in the via. To recover the traction-free boundary condition on the surfaces in the original problem, the normal stress on the surface is removed by superimposing Problem B [Fig. 2(c)], in which a pressure of the same magnitude $(p=$ $\left.\sigma_{z}\right)$ is applied at both ends of the via, but no thermal load. Problem A can be solved analytically, while an approximate solution to Problem B can be obtained semianalytically. The same method was used previously to determine the stress field in fiber-reinforced intermetallic composites [15].

The exact solution to Problem A in Fig. 2(b) is identical to the 2-D plane-strain solution to the classical Lame problem in elasticity [16]. The stress in the via is uniform and triaxial, with the following components:

$$
\begin{gathered}
\sigma_{r}^{A}=\sigma_{\theta}^{A}=\frac{-E_{f} \varepsilon_{T}}{1-2 v_{f}+\frac{1+v_{m}}{1+v_{f}} \frac{E_{f}}{E_{m}}} \\
\sigma_{z}^{A}=-E_{f} \varepsilon_{T}\left[\frac{1+\frac{1+v_{m}}{1+v_{f}} \frac{E_{f}}{E_{m}}}{1-2 v_{f}+\frac{1+v_{m}}{1+v_{f}} \frac{E_{f}}{E_{m}}}\right]
\end{gathered}
$$

where $\sigma_{r}, \sigma_{\theta}$, and $\sigma_{z}$ are the radial, circumferential, and axial stresses, respectively, and $\varepsilon_{T}=\left(\alpha_{f}-\alpha_{m}\right) \Delta T$ is the mismatch strain due to a thermal load $\Delta T$. The material properties $\alpha$,
$E$, and $\nu$ are the CTE, Young's modulus, and Poisson's ratio, with the subscripts $f$ and $m$ for the via (fiber) and Si (matrix), respectively. In contrast, the corresponding stress field in $\mathrm{Si}$ $\left(r>D_{f} / 2\right)$ is nonuniform and biaxial

$$
\sigma_{r}^{A}=-\sigma_{\theta}^{A}=\frac{-E_{f} \varepsilon_{T}}{1-2 v_{f}+\frac{1+v_{m}}{1+v_{f}} \frac{E_{f}}{E_{m}}}\left(\frac{D_{f}}{2 r}\right)^{2}
$$

where $D_{f}$ is the diameter of the TSV and $r$ is the radial coordinate measured from the center of the via. The stress field in (1)-(3) can be simplified by neglecting the elastic mismatch between the via and $\mathrm{Si}$, with $E_{f}=E_{m}=E$ and $v_{f}=v_{m}=v$ as given in the previous studies [13], [14].

The aforementioned 2-D solution does not satisfy the traction-free boundary condition on the surfaces in the original problem [Fig. 2(a)] because of the presence of the axial stress $\left(\sigma_{z}^{A}\right)$ in the via. This is corrected by superimposing Problem B in Fig. 2(c), with a uniform axial stress of the same magnitude acting at both ends of the TSV in the opposite direction (i.e., $p=\sigma_{z}^{A}$ ). The stress field due to the surface pressure is typically localized near the ends of the via. Thus, the stress distribution from the 2-D solution in (1)-(3) is an accurate solution at locations far away from the ends of the TSV, particularly for high-aspect-ratio (height/diameter or $H / D_{f}$ ) TSVs embedded in a thick wafer. However, the correction due to Problem B renders a very different stress distribution near the wafer surface around the TSV. For a relatively thin wafer, the stress in the entire via and its surrounding can be affected and thus different from the 2-D solution. In the following, we first develop a semianalytical solution to Problem B for a thick wafer and then study the effect of wafer thickness by FEA.

Focusing on the near-surface stress field for Problem B in Fig. 2(c), we consider a semi-infinite wafer subject to a uniform pressure on the surface over a circular area of diameter $D_{f}$. For simplicity, we neglect the elastic mismatch between the via and $\mathrm{Si}$, so that $p=\sigma_{z}^{A}=-E \varepsilon_{T} /(1-\nu)$. Consequently, the solution can be obtained in an integral form based on the 3-D solution to the classical Boussinesq problem in elasticity [16], [17], with the following stress components:

$$
\begin{aligned}
\sigma_{z}^{B}(r, z) & =\frac{E \varepsilon_{T}}{1-\nu} \int_{0}^{2 \pi} \int_{0}^{\frac{D_{f}}{2}} \frac{3 z^{3} \rho d \rho d \theta}{2 \pi R^{5}} \\
\sigma_{r z}^{B}(r, z) & =\frac{E \varepsilon_{T}}{1-\nu} \int_{0}^{2 \pi} \int_{0}^{\frac{D_{f}}{2}} \frac{3 z^{2}(r-\rho \cos \theta) \rho d \rho d \theta}{2 \pi R^{5}}
\end{aligned}
$$




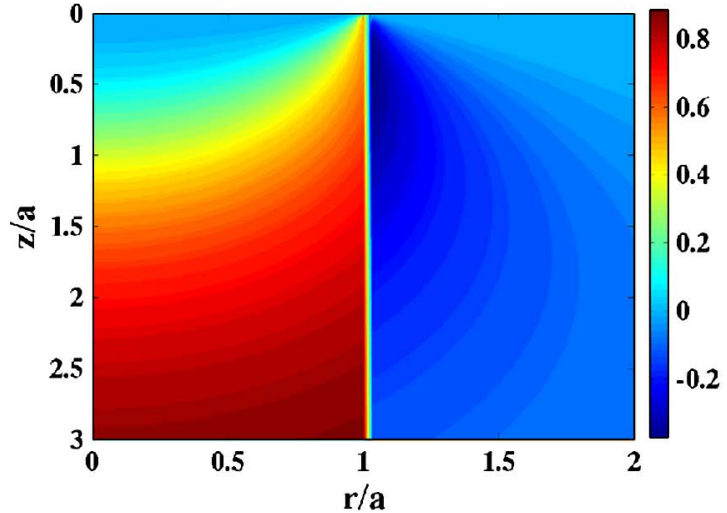

(a)

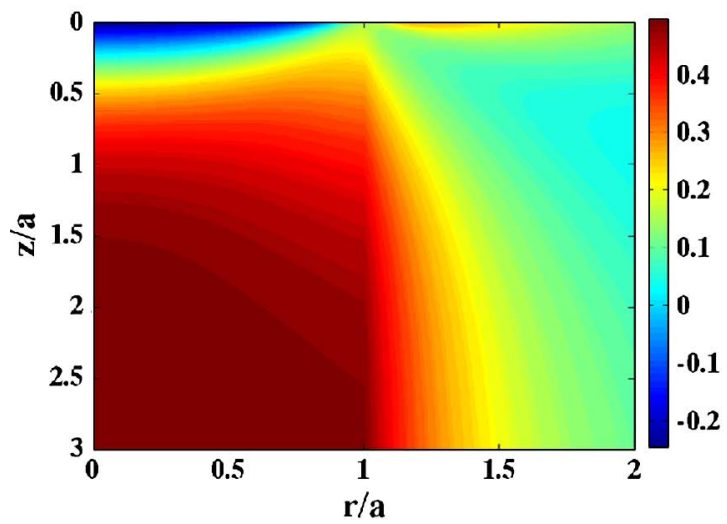

(c)

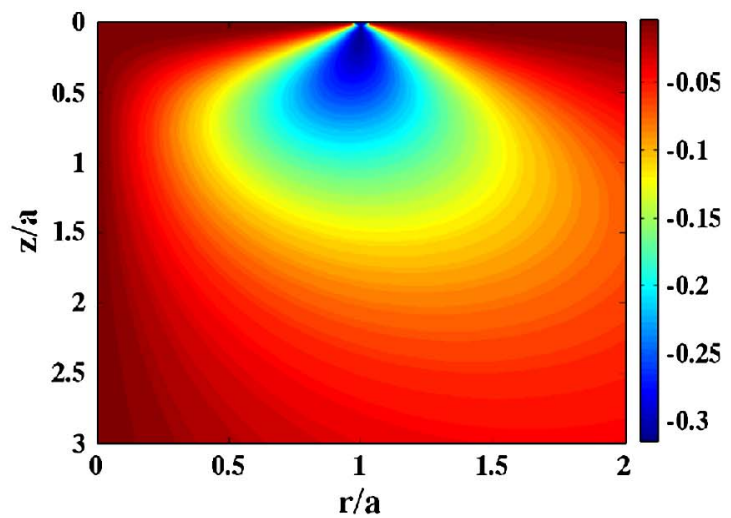

(b)

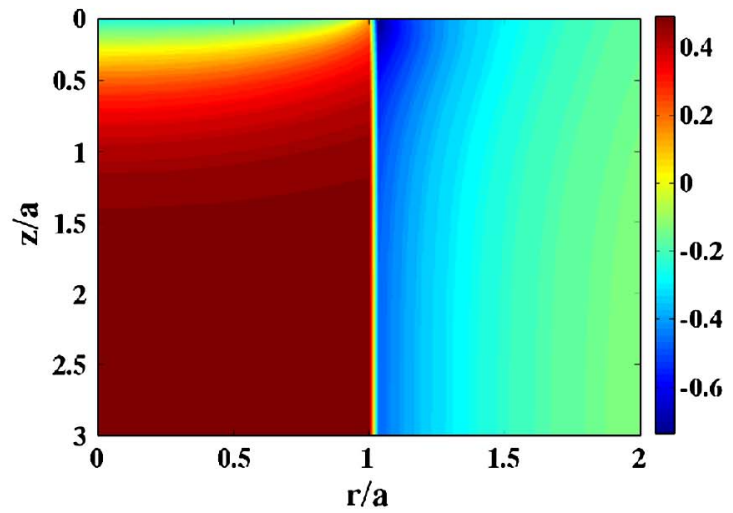

(d)

Fig. 3. Near-surface stress distributions predicted by the semianalytical solution. The stress magnitudes are normalized by $p=-E \varepsilon_{T} /(1-\nu)$, and $r$ is normalized by the via radius $\left(a=D_{f} / 2\right)$. (a) Axial stress $\left(\sigma_{z}\right)$. (b) Shear stress $\left(\sigma_{r z}\right)$. (c) Radial stress $\left(\sigma_{r}\right)$. (d) Circumferential stress $\left(\sigma_{\theta}\right)$.

$$
\begin{aligned}
\sigma_{r}^{B}(r, z)= & \frac{-E \varepsilon_{T}}{2 \pi(1-\nu)} \\
& \quad \times \int_{0}^{\frac{D_{f}}{2}} \int_{0}^{2 \pi}\left[\left(\frac{1-2 v}{R^{2}+R z}-\frac{3 z\left(R^{2}-z^{2}\right)}{R^{5}}\right) \cos ^{2} \beta\right. \\
& \left.\quad+\left(\frac{z}{R^{3}}-\frac{1}{R^{2}+R z}\right)(1-2 \nu) \sin ^{2} \beta\right] \rho d \rho d \theta
\end{aligned}
$$

$$
\begin{aligned}
\sigma_{\theta}^{B}(r, z)= & \frac{-E \varepsilon_{T}}{2 \pi(1-\nu)} \\
& \quad \times \int_{0}^{\frac{D_{f}}{2}} \int_{0}^{2 \pi}\left[\left(\frac{1-2 v}{R^{2}+z R}-\frac{3 z\left(R^{2}-z^{2}\right)}{R^{5}}\right) \sin ^{2} \beta\right. \\
& \left.\quad+\left(\frac{z}{R^{3}}-\frac{1}{R^{2}+z R}\right)(1-2 \nu) \cos ^{2} \beta\right] \rho d \rho d \theta
\end{aligned}
$$

where $\quad R=\sqrt{z^{2}+\rho^{2}+r^{2}-2 \rho r \cos \theta} \quad$ and $\quad \beta=$ $\tan ^{-1}[\rho \sin \theta /(r-\rho \cos \theta)]$. The stress field is axisymmetric, varying with both $r$ and $z$ in the cylindrical coordinate with $z=0$ at the surface.

Finally, the near-surface stress distribution around the via is obtained by adding the stress distributions in (4)-(7) onto (1)-(3) for both the via and the Si wafer, i.e., $\sigma_{z}=\sigma_{z}^{A}+\sigma_{z}^{B}$, etc. The contours of the overall stress distribution are shown in Fig. 3, where the stress magnitude is normalized by the pressure $p=-E \varepsilon_{T} /(1-\nu)$, and the integrals in (4)-(7) are evaluated numerically by the method of quadratic interpolation [18]. Fig. 3(a) shows that the normal stress $\sigma_{z}$ is zero on the surface $(z=0)$, as required by the traction-free boundary condition. The normal stress is nonuniform in the via and Si near the surface. Unlike the 2-D solution, the shear stress $\left(\sigma_{z r}\right)$ is not zero near the end of the via [Fig. 3(b)]. In fact, a concentration of the shear stress is predicted at the junction between the surface $(z=0)$ and via/Si interface $\left(r=D_{f} / 2\right)$, which can contribute to the driving force to cause interfacial delamination. The distributions of the radial stress $\left(\sigma_{r}\right)$ and the circumferential stress $\left(\sigma_{\theta}\right)$ near the end of TSV [Fig. 3(c) and (d)] are also very different from the predictions by the 2-D solution. Depending on the sign of the thermal mismatch strain, $\varepsilon_{T}=\left(\alpha_{f}-\alpha_{m}\right) \Delta T$, the stresses can be either tensile or compressive. For example, if $\alpha_{f}>\alpha_{m}, p<0$ for heating $(\Delta T>0)$ and $p>0$ for cooling $(\Delta T<0)$. For the case of cooling, the radial stress is tensile along the via/Si interface, which can contribute to the driving force for interfacial delamination. The radial stress is also tensile in $\mathrm{Si}$ near the surface, which may cause circumferential cracking (C-cracks) of the Si. During heating, the circumferential stress is tensile in $\mathrm{Si}$, which may cause radial cracks (R-cracks) in $\mathrm{Si}$. For both heating and cooling, the presence of the shear stress $\left(\sigma_{r z}\right)$ along the TSV/Si interface can induce interfacial failure by delamination. In this paper, we focus on interfacial delamination as the critical failure mode under both heating and cooling conditions. 
At the center of the via $(r=0)$, the stresses can be obtained in closed form as follows [16]:

$$
\begin{aligned}
\sigma_{z}(z ; r=0)= & -\frac{E \varepsilon_{T}}{1-v} \frac{z^{3}}{\left(z^{2}+\frac{D_{f}^{2}}{4}\right)^{\frac{3}{2}}} \\
\sigma_{r}(z ; r=0)= & \sigma_{\theta}(z ; r=0) \\
= & -\frac{E \varepsilon_{T}}{2(1-v)} \\
& \times\left[-2 \nu+\frac{2(1+\nu) z}{\left(z^{2}+\frac{D_{f}^{2}}{4}\right)^{\frac{1}{2}}}-\frac{z^{3}}{\left(z^{2}+\frac{D_{f}^{2}}{4}\right)^{\frac{3}{2}}}\right] .
\end{aligned}
$$

The variation of the stresses in the via is important for the study of plastic yielding and stress migration in TSVs.

On the Si surface $\left(z=0\right.$ and $\left.r>D_{f} / 2\right)$, it is found that $\sigma_{r}+\sigma_{\theta}=0$, which suggests weak Raman shifts for stress measurements using scanning micro-Raman spectroscopy [19]. However, consideration of the elastic anisotropy of Si would yield a nonzero sum of the stresses. It is also important to recognize the variation of the near-surface stresses in the $z$-direction, since the Raman measurement typically averages over certain depth below the surface. Depending on the magnitudes and signs of the stresses, channeling cracks may grow at the $\mathrm{Si}$ surface near the TSV, in either the radial or the circumferential direction. The near-surface stresses can also degrade the electrical performance of the devices located near the Si surface and the TSV [8]. Thus, understanding the characteristics of the near-surface stresses in $\mathrm{Si}$ is essential for the design of the keepaway zone [9], [14] around the TSV to mitigate the impact of stresses on the device performance.

\section{B. FEA}

To verify the semianalytic solution developed in the previous section, FEA is performed using the commercial package ABAQUS (v6.8). Since the thickness of the Si wafer is one of the key design parameters for the TSV structure, the effect of wafer thickness on thermal stress distribution is examined by FEA models with two different thicknesses. The model structure is shown in Fig. 1(a), with the TSV diameter $D_{f}=$ $30 \mu \mathrm{m}$ and the wafer thickness $H=300$ and $60 \mu \mathrm{m}$. A negative thermal loading (cooling), $\Delta T=-250{ }^{\circ} \mathrm{C}$, is assumed. The material properties are $E_{f}=E_{m}=110 \mathrm{GPa}, v_{f}=v_{m}=$ $0.35, \alpha_{f}=17 \mathrm{ppm} /{ }^{\circ} \mathrm{C}$, and $\alpha_{m}=2.3 \mathrm{ppm} /{ }^{\circ} \mathrm{C}$. The model is an approximation to a $\mathrm{Cu}$ TSV in $\mathrm{Si}$, neglecting the elastic mismatch between $\mathrm{Cu}$ and $\mathrm{Si}$. In practice, a thin barrier layer is typically needed between the $\mathrm{Cu}$ via and $\mathrm{Si}$, which has minimal effects on the stress distribution and is thus ignored here.

Fig. 4 shows the FEA results, in comparison with the semianalytical solution. First, the axial stress $\left(\sigma_{z}\right)$ along the center line of the TSV $(r=0)$ shows the transition from zero stress at the surface $(z=0)$ to a tensile stress away from the surface [Fig. 4(a)]. For the thick wafer $\left(H / D_{f}=10\right)$, the FEA result shows excellent agreement with the analytical solution in (8), both approaching the 2-D solution (the dashed line) away from the surface. For the thin wafer $\left(H / D_{f}=2\right)$, however, the axial stress in the TSV is significantly lower, due to close proximity

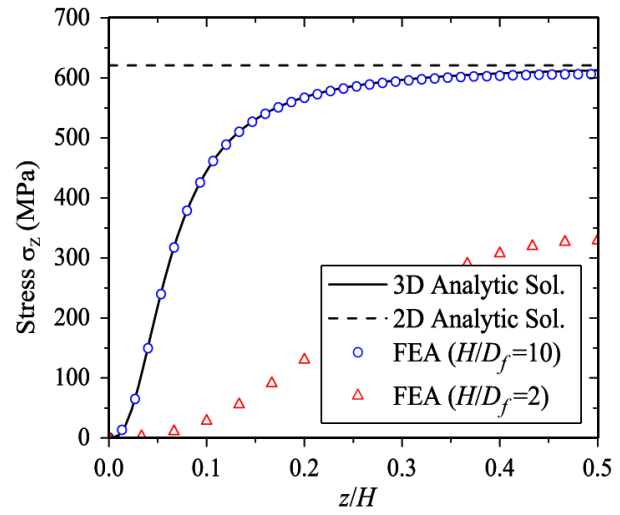

(a)

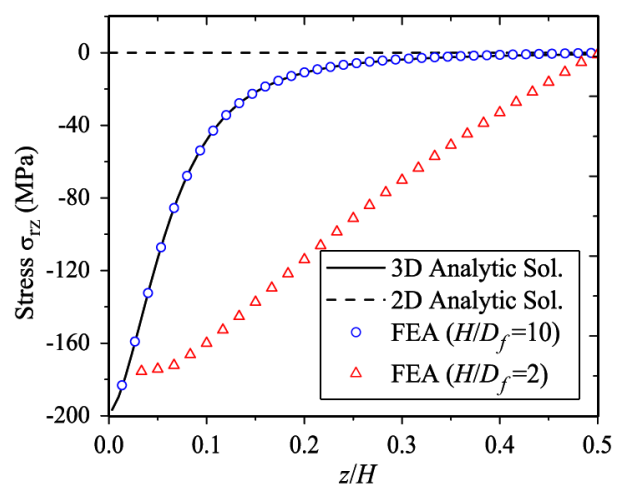

(b)

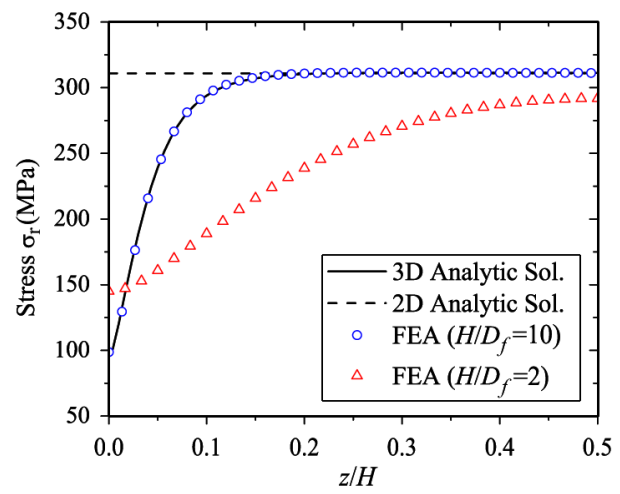

(c)

Fig. 4. Effect of wafer thickness on stress distributions $\left(D_{f}=30 \mu \mathrm{m}\right.$ and $\left.\Delta T=-250{ }^{\circ} \mathrm{C}\right)$. (a) Axial stress at the via center $(r=0)$. (b) Shear stress at the TSV/Si interface $\left(r=D_{f} / 2\right)$. (c) Radial stress at the TSV/Si interface $\left(r=D_{f} / 2\right)$.

of the two free surfaces. The shear and radial stresses along the TSV/Si interface $\left(r=D_{f} / 2\right)$ are shown in Fig. 4(b) and (c), respectively. Again, the semianalytical solution compares closely with the FEA results for the thick wafer. By symmetry, the shear stress is zero at the midplane of the wafer $(z / H=$ $0.5)$. Based on an asymptotic analysis of the semianalytical solution [17], the magnitude of the shear stress at the interface approaches a finite value $\left(\sigma_{r z} \rightarrow-p / \pi\right)$ at the surface $(z=0)$. In between, the variation of the shear stress depends on the wafer thickness. Similarly, the radial stress $\left(\sigma_{r}\right)$ at the interface asymptotically approaches a finite value $\left(\sigma_{r} \rightarrow(0.5-\nu) p\right)$ at $z=0$ and approaches the 2-D solution (the dashed line, $\sigma_{r} \rightarrow$ $0.5 p$ ) far away from the surface. For the thinner wafer, the radial stress is slightly higher near the surface but is lower elsewhere. 


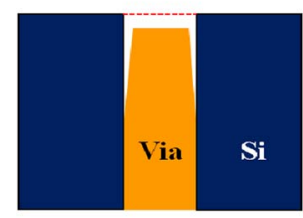

Mode I+ II

(a)

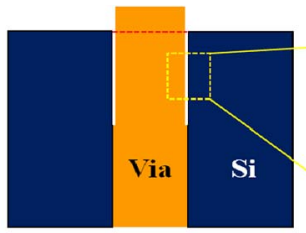

Mode II

(b)

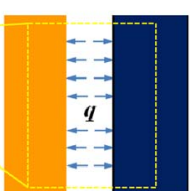

Contact force, $q$
Fig. 5. Schematics of interfacial delamination of TSV under cooling and heating conditions. In both cases, the interfacial crack is assumed to grow axisymmetrically from one surface toward the other surface. (a) Cooling $(\Delta T<0)$. (b) Heating $(\Delta T>0)$.

In the case of cooling $(\Delta T<0)$, both the shear stress and the tensile radial stress contribute to the driving force for interfacial delamination. The effect of wafer thickness on the delamination driving force will be analyzed in the next section. It is seen from Fig. 4 that the 2-D plane-strain solution only predicts stresses far away from the wafer surface, while the semianalytical 3-D solution is a good approximation everywhere for relatively thick wafers (e.g., $H / D_{f}>10$ ). Neither solution is applicable for relatively thin wafers.

\section{ANALYSis OF INTERFACIAL DELAMINATION}

The stress analysis in the previous section suggests a potential failure mechanism of the TSV structure due to interfacial delamination. Fig. 5 shows two modes of interfacial delamination for a fully filled TSV structure. With a negative thermal load $(\Delta T<0)$, the radial stress along the via/Si interface is tensile (assuming $\alpha_{f}>\alpha_{m}$ ). Consequently, the interfacial delamination crack may grow in a mixed mode (peeling and shearing). With a positive thermal load $(\Delta T>0)$, however, the radial stress is compressive, which does not contribute to the driving force for delamination. This results in an interfacial crack with a pure shearing mode (mode II). In this case [Fig. 5(b)], the two crack faces are in contact and may be subject to friction. For simplicity, we assume a frictionless contact in this paper. In the following, we first develop analytical solutions for the steady-state energy release rate of the interfacial crack, under both cooling and heating conditions. The analytical solutions are then compared to FEA, which is extended to study the effects of crack length and wafer thickness on the fracture driving force.

\section{A. Steady-State Delamination: Cooling Versus Heating}

For a TSV with a relatively high aspect ratio $\left(H / D_{f}\right)$, the energy release rate for interfacial delamination reaches a steady state when the crack length is several times greater than the via diameter. Since the energy release rate is usually lower for shorter cracks, the steady-state value sets an upper bound for the fracture driving force, which may be used as the critical condition for conservative design of reliable TSV structures.

Consider an infinitely long fiber (TSV) in an infinite matrix (Si wafer), with a semi-infinite circumferential crack along the interface and subjected to a thermal load $(\Delta T)$. The steadystate energy release rate for the interfacial crack growth (per unit area) is obtained by comparing the elastic strain energy far ahead of the crack front and that far behind the crack front. While the stress field near the crack front is complicated with singularity and 3-D distribution, it translates in a steady state as the crack front advances. Far ahead of the crack front, the stress field can be obtained analytically by solving the 2-D planestrain problem [Problem A in Fig. 2(b)]. Far behind the crack front, since the TSV is debonded from $\mathrm{Si}$, the stress is relaxed in both the via and Si. For the case of cooling $(\Delta T<0)$, the stress is zero in both TSV and Si. For heating $(\Delta T>0)$, however, the contact between the crack faces induces a stress field similar to Problem A, but the axial stress $\left(\sigma_{z}\right)$ in the via is zero under the assumption of frictionless contact.

Based on (1)-(3), the elastic strain energy densities (per unit volume) in the TSV and Si far ahead of the crack front are, respectively

$$
\begin{aligned}
& U_{f}^{A}=\frac{1}{2} E_{f} \varepsilon_{T}^{2}\left[1+\frac{2\left(1-2 v_{f}\right)\left(1+v_{f}\right)}{\left(1-2 v_{f}+\frac{1+v_{m}}{1+v_{f}} \frac{E_{f}}{E_{m}}\right)^{2}}\right] \\
& U_{m}^{A}=E_{f} \varepsilon_{T}^{2} \frac{\left(1+v_{m}\right) E_{f} / E_{m}}{\left(1-2 v_{f}+\frac{1+v_{m}}{1+v_{f}} \frac{E_{f}}{E_{m}}\right)^{2}}\left(\frac{D_{f}}{2 r}\right)^{4} .
\end{aligned}
$$

For the case of cooling, the release of elastic energy per unit length of the interfacial crack is

$$
\Delta U_{\text {cooling }}=\frac{\pi}{4} D_{f}^{2} U_{f}^{A}+2 \pi \int_{\frac{D_{f}}{2}}^{\infty} U_{m}^{A}(r) r d r .
$$

The steady-state energy release rate (per unit area) is thus

$$
\begin{aligned}
G_{\text {cooling }}^{\mathrm{SS}}= & \frac{\Delta U_{\text {cooling }}}{\pi D_{f}}=\frac{E_{m} \varepsilon_{T}^{2} D_{f}}{4} \\
& \times\left(\frac{\left(1+v_{f}\right)(1+\alpha)}{\left(1-2 v_{f}\right)(1-\alpha)+(1+\alpha) \frac{1+v_{m}}{1+v_{f}}}+\frac{1}{2} \frac{1+\alpha}{1-\alpha}\right)
\end{aligned}
$$

where $\alpha=\left(\bar{E}_{f}-\bar{E}_{m}\right) /\left(\bar{E}_{f}+\bar{E}_{m}\right)$ is the Dundar's parameter for elastic mismatch between the TSV and Si, with $\bar{E}=$ $E /\left(1-v^{2}\right)$. If the elastic mismatch is neglected (i.e., $\alpha=0$ and $\left.\nu_{f}=\nu_{m}=\nu\right),(13)$ is reduced to a simpler form

$$
G_{\text {cooling }}^{\mathrm{SS}}=\frac{E \varepsilon_{T}^{2} D_{f}}{4(1-\nu)}
$$

Under the heating condition $(\Delta T>0)$, due to the contact of the crack faces [Fig. 5(b)], the stress state in the TSV far behind the crack front is equibiaxial

$$
\sigma_{r}=\sigma_{\theta}=-\frac{E_{f} \varepsilon_{T}}{1-v_{f}+\left(1+v_{m}\right) \frac{E_{f}}{E_{m}}} .
$$

Correspondingly, the stress field in the matrix ( $\mathrm{Si})$ is

$$
\sigma_{r}=-\sigma_{\theta}=-\frac{E_{f} \varepsilon_{T}}{1-v_{f}+\left(1+v_{m}\right) \frac{E_{f}}{E_{m}}} \frac{D_{f}^{2}}{4 r^{2}} .
$$


Thus, the elastic strain energy densities (per unit volume) of the TSV and Si far behind the crack front are, respectively

$$
\begin{aligned}
U_{f}^{C} & =\frac{E_{f} \varepsilon_{T}^{2}\left(1-v_{f}\right)}{\left[1-v_{f}+\left(1+v_{m}\right) \frac{E_{f}}{E_{m}}\right]^{2}} \\
U_{m}^{C} & =E_{f} \varepsilon_{T}^{2} \frac{\left(1+v_{m}\right) E_{f} / E_{m}}{\left[1-v_{f}+\left(1+v_{m}\right) \frac{E_{f}}{E_{m}}\right]^{2}}\left(\frac{D_{f}}{2 r}\right)^{4} .
\end{aligned}
$$

Therefore, the release of the elastic strain energy per unit length of the interfacial crack is

$$
\Delta U_{\text {heating }}=\frac{\pi}{4} D_{f}^{2}\left(U_{f}^{A}-U_{f}^{C}\right)+2 \pi \int_{D_{f} / 2}^{\infty}\left(U_{m}^{A}-U_{m}^{C}\right) r d r .
$$

The steady-state energy release rate for heating is then

$$
\begin{aligned}
G_{\text {heating }}^{\text {SS }}= & \frac{\Delta U_{\text {heating }}}{\pi D_{f}}=\frac{E_{m} \varepsilon_{T}^{2} D_{f}}{4} \\
\times & {\left[\frac{(1+\alpha)\left(1+v_{f}\right)}{(1-\alpha)\left(1-2 v_{f}\right)+(1+\alpha) \frac{1+v_{m}}{1+v_{f}}}+\frac{1}{2} \frac{1+\alpha}{1-\alpha}\right.} \\
& \left.-\frac{(1+\alpha)}{\left(1-v_{f}\right)(1-\alpha)+\left(1+v_{m}\right)(1+\alpha)}\right] .
\end{aligned}
$$

Again, a simpler result can be obtained by neglecting the elastic mismatch, namely

$$
G_{\text {heating }}^{\mathrm{SS}}=\frac{1+v}{8(1-v)} E \varepsilon_{T}^{2} D_{f} .
$$

Several interesting results can be deduced based on the analytical solutions for the steady-state energy release rates. First, the steady-state energy release rate for interfacial delamination is linearly proportional to the TSV diameter, which may set an upper bound for the via diameter in order to avoid delamination. Second, the energy release rate is proportional to the square of the thermal mismatch strain $\varepsilon_{T}=\left(\alpha_{f}-\alpha_{m}\right) \Delta T$. Thus, the delamination driving force can be reduced by either using TSV materials with smaller thermal expansion mismatch $\left(\alpha_{f}-\alpha_{m}\right)$ and/or reducing the thermal loads $(\Delta T)$. Third, the energy release rate for interfacial delamination increases with the elastic modulus of the TSV material; however, the effect is less prominent than the effect of the thermal expansion mismatch. Finally, a comparison between (14) and (21) indicates that, with the same magnitude for the thermal load $(\Delta T)$, the driving force for interfacial delamination under cooling is about twice of that under heating, a result that can be attributed to the presence of the tensile radial stress $\left(\sigma_{r}\right)$ across the interface (opening mode) for the case of cooling. Furthermore, we note that the energy release rate should be compared to the adhesion energy (fracture toughness) of the interface in order to assess the interfacial reliability, as discussed later.

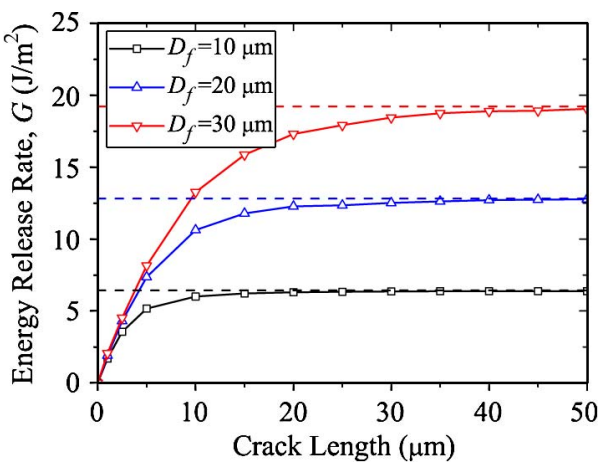

Fig. 6. Effect of crack length on the energy release rate for interfacial delamination of TSVs $\left(H=300 \mu \mathrm{m}\right.$ and $\left.\Delta T=-250^{\circ} \mathrm{C}\right)$.

TABLE I

THERMOMECHANICAL PROPERTIES OF THE MATERIALS USED IN THIS PAPER

\begin{tabular}{cccc}
\hline \hline Material & $\begin{array}{c}\text { CTE } \\
(\mathrm{ppm} / \mathrm{K})\end{array}$ & $\begin{array}{c}\text { Young's Modulus } \\
(\mathrm{GPa})\end{array}$ & Poisson's ratio \\
\hline $\mathrm{Si}$ & 2.3 & 130 & 0.28 \\
$\mathrm{Cu}$ & 17 & 110 & 0.35 \\
$\mathrm{Al}$ & 20 & 70 & 0.35 \\
$\mathrm{Ni}$ & 13 & 207 & 0.31 \\
$\mathrm{~W}$ & 4.4 & 400 & 0.28 \\
$\mathrm{BCB}$ & 40 & 3.0 & 0.34 \\
\hline \hline
\end{tabular}

\section{B. Effects of Crack Length and Wafer Thickness}

For a finite-sized TSV structure with a finite interfacial crack, the energy release rate depends on both the crack length and the wafer thickness. In Fig. 6, we show the energy release rate as a function of the crack length for different TSV diameters with a wafer thickness $H=300 \mu \mathrm{m}$. The material properties for the via and the substrate are taken as those of $\mathrm{Cu}$ and $\mathrm{Si}$ in Table I. A FEA model of the TSV structure is constructed for each via diameter, and the energy release rates are calculated by the method of J-integral in ABAQUS. As expected, the energy release rate increases with the crack length and approaches the steady-state solution when the crack length is about two to three times the via diameter. By comparing the energy release rate to the interfacial adhesion energy, i.e., $G\left(a_{c}\right)=\Gamma$, a critical crack length $\left(a_{c}\right)$ may be determined, beyond which the delamination crack grows unstably. For a conservative design, one may require $G_{\mathrm{SS}} \leq \Gamma$ so that all cracks remain stable under the prescribed thermal load.

To illustrate the effect of wafer thickness, Fig. 7 shows the energy release rate as a function of the crack length for different wafer thicknesses, with the same via diameter and thermal load. For a relatively thin wafer, the energy release rate for interfacial delamination reaches a maximum and then decreases as the crack length increases, approaching the wafer surface on the other side. The maximum energy release rate decreases as the wafer thickness decreases. Therefore, the interfacial reliability of TSVs may be improved by using thinner wafers, although other effects such as wafer handling and Si cracking may impose a lower limit for the wafer thickness. 


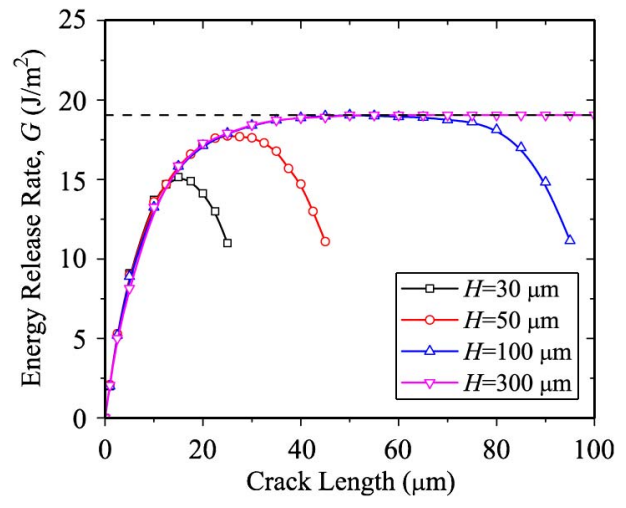

Fig. 7. Effect of wafer thickness on the energy release rate for interfacial delamination of TSVs $\left(D_{f}=30 \mu \mathrm{m}\right.$ and $\left.\Delta T=-250^{\circ} \mathrm{C}\right)$.

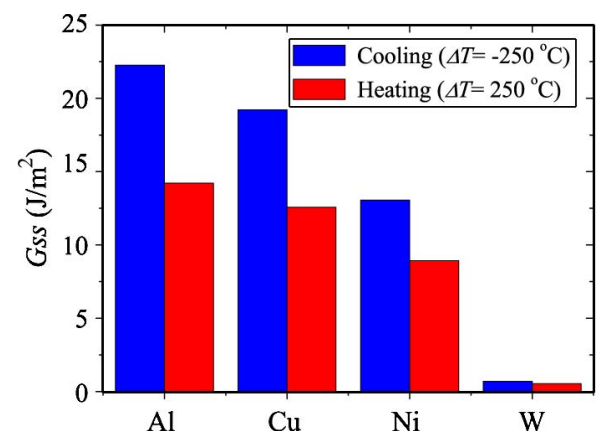

Fig. 8. Comparison of the steady-state energy release rates for interfacial delamination in TSV structures using different via materials, under the same thermal load for cooling and heating $\left(D_{f}=30 \mu \mathrm{m}\right)$.

\section{Discussions}

\section{A. Effects of TSV Materials}

Several other metals, including aluminum (Al), nickel (Ni), and tungsten $(\mathrm{W})$, have been considered to replace $\mathrm{Cu}$ as alternative materials for TSVs. The effect of materials on interfacial fracture driving force for the fully filled TSVs is evaluated using the thermomechanical properties listed in Table I. The steady-state energy release rates for the four TSV materials are compared in Fig. 8 under both cooling $\left(\Delta T=-250{ }^{\circ} \mathrm{C}\right)$ and heating $\left(\Delta T=250{ }^{\circ} \mathrm{C}\right)$ conditions, with the same TSV diameter of $30 \mu \mathrm{m}$. Compared to $\mathrm{Cu}, \mathrm{Al}$ has a lower Young's modulus but a larger mismatch in CTE with Si. Consequently, the driving force for interfacial delamination is higher for $\mathrm{Al}$ under the same thermal load. In contrast, $\mathrm{Ni}$ has a higher Young's modulus than $\mathrm{Cu}$ but a lower thermal mismatch, resulting in a lower driving force for delamination. Despite the highest Young's modulus, W has a very small CTE mismatch with $\mathrm{Si}$, and thus, the delamination driving force is significantly lower than that for the Cu TSV. This renders W a particularly attractive material for TSV applications from the interfacial reliability perspective.

To evaluate the material effect on the TSV reliability, other factors have to be considered in addition to the fracture driving force. In particular, these include the processing temperature for the specific metal, the TSV diameter, and the interfacial adhesion energy. Since different TSV materials may require processes with different thermal loads, this can affect the delamination driving force, which is proportional to the square of the thermal mismatch strain. Among the four metals considered here, the CVD process for $\mathrm{W}$ deposition has the highest temperature at around $400{ }^{\circ} \mathrm{C}$ and, thus, the highest thermal load. This is balanced by the relatively small diameter for the W TSV. For each TSV material, the energy release rate has to be compared with the specific interfacial adhesion energy, i.e., $G\left(a_{c}\right)=\Gamma$, to determine a critical crack length $\left(a_{c}\right)$, beyond which the delamination crack grows unstably. The interfacial adhesion varies with the TSV material and may be enhanced by using thin adhesive barrier layers between the TSV and Si [20]. In addition, plastic deformation of the TSV metals has not been considered in this paper. Plastic yielding could partly relax the thermal stress in the via and the $\mathrm{Si}$, thus reducing the fracture driving force. Moreover, the energy dissipation during plastic deformation could contribute to the overall fracture energy [21]. Therefore, the interfacial reliability may be improved by plasticity in the via. However, plastic deformation is irrecoverable and could lead to other reliability issues such as dislocations, stress voiding, and fatigue. Further studies are required to understand the effect of plasticity on thermomechanical reliability of TSVs.

\section{B. Effects of Dielectric Buffer Layer}

This paper has considered a much simplified structure with a single TSV embedded in $\mathrm{Si}$. In practice, a thin barrier layer and/or a dielectric buffer layer may be needed between the TSV and Si. For example, to fabricate Cu TSVs, a dielectric or a nitride barrier layer is typically deposited on the via sidewall before $\mathrm{Cu}$ electroplating. The dielectric layer, which is often made of silicon dioxide with $1-2-\mu \mathrm{m}$ thickness, provides insulation of the TSV from the silicon substrate. Similar to the $\mathrm{Cu}$ damascene interconnects, the barrier layer is usually made of metallic materials such as Ti, Ta, and their respective nitrides, TiN and TaN, with thickness less than $0.1 \mu \mathrm{m}$ [22]-[24]. The relatively thin barrier layer has little effect on the thermal stresses and the interfacial fracture driving force, but it may play an important role by enhancing the interfacial adhesion [20]. On the other hand, the much thicker dielectric buffer layer could serve as a stress buffer to reduce the thermal expansion mismatch between the TSV and $\mathrm{Si}$, thus reducing the thermal stress and the fracture driving force. For this purpose, polymeric materials such as Parylene and Benzocyclobutene (BCB) have been used recently to replace the oxide layer [25], [26]. By using a 2-5- $\mu \mathrm{m}$-thick polymer buffer layer, the thermal stress in the TSV structure can be considerably reduced [13], and the electrical performance can be improved by reducing the capacitive coupling [26].

As an example, we consider a Cu TSV of diameter $30 \mu \mathrm{m}$ with a 5- $\mu \mathrm{m}$ BCB buffer layer [Fig. 1(b)]. The radial and shear stresses along the $\mathrm{Cu} / \mathrm{BCB}$ and $\mathrm{Si} / \mathrm{BCB}$ interfaces are shown in Fig. 9(a) and (b), respectively. Compared to the $\mathrm{Cu} / \mathrm{Si}$ interface without the buffer layer, both the radial and the shear stresses are significantly reduced. Fig. 9(c) shows the energy release rates versus the crack length for delamination along the two interfaces, in comparison to the $\mathrm{Cu} / \mathrm{Si}$ interface without the buffer layer. While the energy release rate for the $\mathrm{Cu} / \mathrm{BCB}$ 


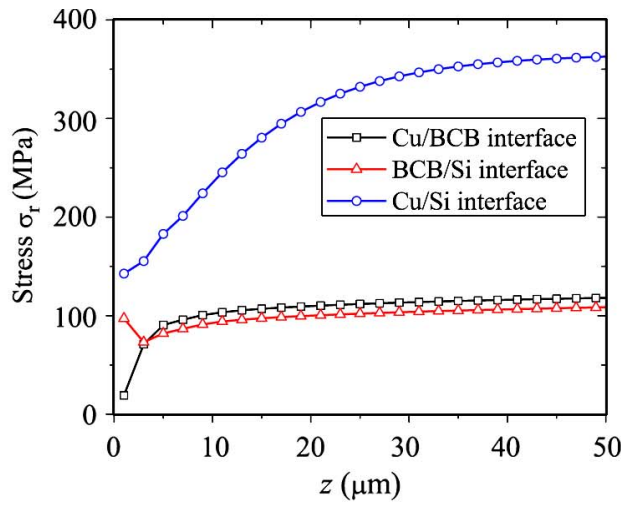

(a)

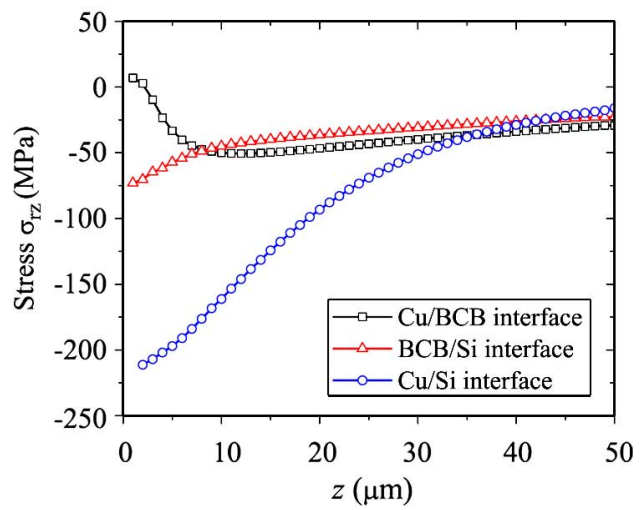

(b)

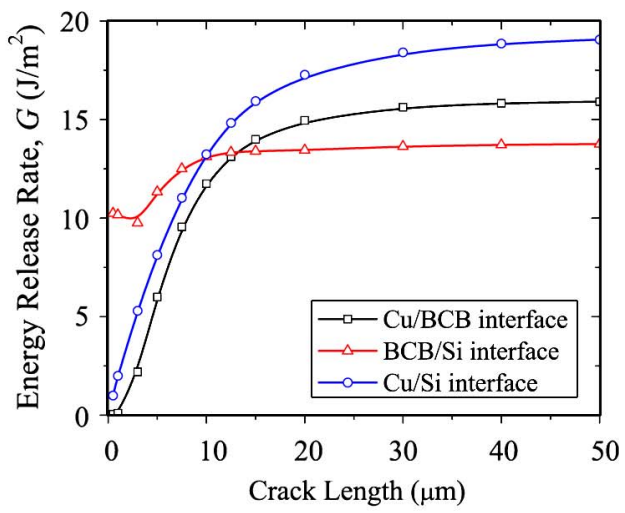

(c)

Fig. 9. Effects of a BCB buffer layer. (a) Radial stress and (b) shear stress along the interfaces. (c) Energy release rate for interfacial delamination $\left(D_{f}=\right.$ $30 \mu \mathrm{m}, t_{b}=5 \mu \mathrm{m}, H=300 \mu \mathrm{m}$, and $\left.\Delta T=-250^{\circ} \mathrm{C}\right)$.

interface is consistently lower than that of the $\mathrm{Cu} / \mathrm{Si}$ interface, the energy release rate for the $\mathrm{Si} / \mathrm{BCB}$ interface is surprisingly high at the limit of short cracks. As shown in a previous study [27], the asymptotic behavior for the energy release rate of a short interfacial crack depends on the elastic mismatch of the two materials. Previous measurements have reported several interfacial fracture energies [28], which are $12.2 \mathrm{~J} / \mathrm{m}^{2}$ for the $\mathrm{Cu} / \mathrm{BCB}$ interface and over $24 \mathrm{~J} / \mathrm{m}^{2}$ for the $\mathrm{Si} / \mathrm{BCB}$ interface. A comparison between the energy release rates in Fig. 9(c) and the respective fracture energy values suggests that delamination is more likely to occur along the $\mathrm{Cu} / \mathrm{BCB}$ interface. To reduce the energy release rate, the material and the via structure will have to be further optimized, for example, by reducing the via diameter or by introducing an adhesive layer at the via/BCB interface.

\section{SUMMARY}

In this paper, the characteristics of thermal stresses in a TSV structure have been analyzed by a semianalytical approach and FEA calculations. It has been emphasized that the 3-D near-surface stress distributions are dramatically different from the analytical solution based on a simple 2-D model. The energy release rate for interfacial delamination of TSV has been evaluated under both cooling and heating conditions, with analytical solutions under the steady-state condition and numerical solutions by FEA models. Based on these results, the effects of the TSV dimensions (e.g., via diameter and wafer thickness) on the interfacial reliability have been elucidated. Furthermore, the effects of via material properties and dielectric buffer layers have been discussed. Together, the potential of materials and structure optimization for improving TSV reliability is envisaged as the key for the development of 3-D interconnects.

\section{ACKNOWLEDGMENT}

The authors would like to thank Dr. P. Garrou, Dr. M. Lane, and Dr. J. Liu for their helpful suggestions and discussions.

\section{REFERENCES}

[1] International Technology Roadmap for Semiconductors (ITRS), 2009

[2] M. S. Bakir and J. D. Meindl, Integrated Interconnect Technologies for 3D Nanoelectronic Systems. Norwood, MA: Artech House, 2009.

[3] J. U. Knickerbocker, P. S. Andry, B. Dang, R. R. Horton, M. J. Interrante, C. S. Patel, R. J. Polastre, K. Sakuma, R. Sirdeshmukh, E. J. Sprogis, S. M. Sri-Jayantha, A. M. Stephens, A. W. Topol, C. K. Tsang, B. C. Webb, and S. L. Wright, "Three-dimensional silicon integration," IBM J. Res. Develop., vol. 52, no. 6, pp. 553-569, Nov. 2008.

[4] J.-Q. Lu, "3-D hyperintegration and packaging technologies for micronano systems," Proc. IEEE, vol. 97, no. 1, pp. 18-30, Jan. 2009.

[5] L. W. Schaper, S. L. Burkett, S. Spiesshoefer, G. V. Vangara, Z. Rahman, and S. Polamreddy, "Architectural implications and process development of 3-D VLSI Z-axis interconnects using through silicon vias," IEEE Trans. Adv. Packag., vol. 28, no. 3, pp. 356-366, Aug. 2005.

[6] K. Sakuma, P. S. Andry, C. K. Tsang, S. L. Wright, B. Dang, C. S. Patel, B. C. Webb, J. Maria, E. J. Sprogis, S. K. Kang, R. J. Polastre, R. R. Horton, and J. U. Knickerbocker, "3D chip-stacking technology with through-silicon vias and low-volume lead-free interconnections," IBM J. Res. Develop., vol. 52, no. 6, pp. 611-622, Nov. 2008.

[7] K. Sakuma, N. Nagai, M. Saito, J. Mizuno, and S. Shoji, "Simplified $20-\mu \mathrm{m}$ pitch vertical interconnection process for 3D chip stacking," IEEJ Trans. Elect. Electron. Eng., vol. 4, no. 3, pp. 339-344, May 2009.

[8] S. E. Thompson, G. Sun, Y. Choi, and T. Nishida, "Uniaxial-processinduced strained-Si: Extending the CMOS roadmap," IEEE Trans. Electron Devices, vol. 53, no. 5, pp. 1010-1020, May 2006.

[9] A. P. Karmarker, X. Xu, and V. Moroz, "Performance and reliability analysis of 3D-integration structures employing through silicon via (TSV)," in Proc. IEEE 47th Annu. Int. Reliab. Phys. Symp., Montreal, QC, Canada, 2009, pp. 682-687.

[10] C. S. Selvanayagam, J. H. Lau, X. Zhang, S. K. W. Seah, K. Vaidyanathan, and T. C. Chai, "Nonlinear thermal stress/strain analyses of copper filled TSV (through silicon via) and their flip-chip microbumps," in Proc. ECTC, 2008, pp. 1073-1081.

[11] N. Ranganathan, K. Prasad, N. Balasubramanian, and K. L. Pey, "A study of thermo-mechanical stress and its impact on through-silicon vias," J. Micromech. Microeng., vol. 18, no. 7, p. 075 018, Jun. 2008.

[12] X. Liu, Q. Chen, P. Dixit, R. Chatterjee, R. Tummala, and S. Sitaraman, "Failure mechanisms and optimum design for electroplated copper through-silicon vias (TSV)," in Proc. ECTC, 2009, pp. 624-629. 
[13] K. Lu, X. Zhang, S. Ryu, J. Im, R. Huang, and P. S. Ho, "Thermomechanical reliability of 3-D ICs containing through silicon vias," in Proc. ECTC, 2009, pp. 630-634.

[14] K. Lu, X. Zhang, S. Ryu, R. Huang, and P. S. Ho, "Thermal stresses analysis of 3-D interconnect," in Proc. 10th Int. Workshop Stress-Induced Phenomena Metallization, vol. 1143, AIP Conference, 2009, pp. 224-230.

[15] T. C. Lu, J. Yang, Z. Suo, A. G. Evans, and R. Hecht, "Matrix cracking in intermetallic composites caused by thermal expansion mismatch," Acta Metall. Mater., vol. 39, no. 8, pp. 1883-1890, Aug. 1991.

[16] S. Timoshenko and J. N. Goodier, Theory of Elasticity. New York: McGraw-Hill, 1970, pp. 403-407.

[17] A. E. H. Love, "The stress produced in a semi-infinite solid by pressure on part of the boundary," Philos. Trans. Roy. Soc. London A, Math. Phys. Sci., vol. 228, pp. 377-420, 1929.

[18] A. Horwitz, "A version of Simpson's rule for multiple integrals," $J$. Comput. Appl. Math., vol. 134, no. 1/2, pp. 1-11, Sep. 2001.

[19] R. E. Geer, "Profiling of process-induced stress in $\mathrm{Cu}$ through-silicon vias (TSVs) for wafer-scale, 3D integration," in Proc. 11th Int. Workshop Stress-Induced Phenomena Metallization, Dresden, Germany, Apr. 12-14, 2010.

[20] M. W. Lane, R. H. Dauskardt, N. Krishna, and I. Hashim, "Adhesion and reliability of copper interconnects with Ta and TaN barrier layers," J. Mater. Res., vol. 15, no. 1, pp. 203-211, Jan. 2000.

[21] M. W. Lane, A. Vainchtein, H. Gao, and R. H. Dauskardt, "Plasticity contributions to interface adhesion in thin-film interconnect structures," J. Mater. Res., vol. 15, no. 12, pp. 2758-2769, Dec. 2000.

[22] O. Luhn, C. V. Hoof, W. Ruythooren, and J. Celis, "Barrier and seed layer coverage in 3D structures with different aspect ratios using sputtering and ALD processes," Microelectron. Eng., vol. 85, no. 10, pp. 1947-1951, Oct. 2008

[23] G. Druais, G. Dilliway, P. Fischer, E. Guidotti, A. Radisic, and S. Zahraoui, "High aspect ratio via metallization for 3D integration using CVD TiN barrier and electrografted Cu seed," Microelectron. Eng., vol. 85, no. 10, pp. 1957-1961, Oct. 2008.

[24] S. Lee, R. Hon, S. Zhang, and C. Wong, "3D stacked flip chip packaging with through silicon vias and copper plating or conductive adhesive filling," in Proc. ECTC, 2005, pp. 795-801.

[25] B. Majeed, N. Pham, D. Tezcan, and E. Beyne, "Parylene N as a dielectric material for through silicon vias," in Proc. ECTC, 2008, pp. 1556-1561.

[26] D. Tezcan, F. Duval, H. Philipsen, O. Luhn, P. Soussan, and B. Swinnen, "Scalable through silicon via with polymer deep trench isolation for 3D wafer level packaging," in Proc. ECTC, 2009, pp. 1159-1164.

[27] H. Mei, Y. Pang, and R. Huang, "Influence of interfacial delamination on channel cracking of elastic thin films," Int. J. Fract., vol. 148, no. 4, pp. 331-342, 2007.

[28] J. Im, E. O. , II, J. Theodore Stokich, A. Strandjord, J. Hetzner, J. Curphy, and C. Karas, "On the mechanical reliability of photo-BCB-based thin film dielectric polymer for electronic packaging applications," J. Electron. Packag., vol. 122, no. 1, pp. 28-33, Mar. 2000.

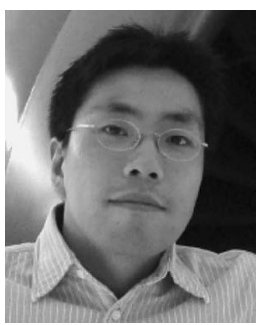

Suk-Kyu Ryu received the B.S. and M.S. degrees in aerospace engineering from Korea Aerospace University, Goyang, Korea, in 1998 and 2000, respectively. He is currently working toward the Ph.D. degree in the Department of Aerospace Engineering and Engineering Mechanics, University of Texas, Austin

He had several years of working experience in composite material industry and in a microsystem laboratory of Korea Institute of Science and Technology. His research focuses on thermomechanical reliability of advanced interconnects and packaging in micro/nanoelectronics.
Kuan-Hsun Lu, photograph and biography not available at the time of publication.

Xuefeng Zhang, photograph and biography not available at the time of publication.

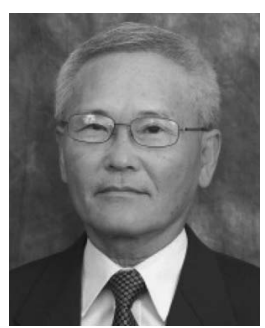

Jang-Hi Im (SM'04) received the B.S. degree in mechanical engineering from Seoul National University, Seoul, Korea, in 1964 and the M.S. degree in mechanical engineering and the Ph.D. degree in materials science and engineering from the Massachusetts Institute of Technology, Cambridge, in 1971 and 1976, respectively.

$\mathrm{He}$ is currently a Research Professor with the Laboratory for Interconnect and Packaging, The University of Texas, Austin. Up until 2004, he was with The Dow Chemical Company for 28 years, taking on various $R \& D$ positions, including Research Scientist in electronic materials. In this last capacity, he had headed materials science and adhesion efforts for $\mathrm{BCB}$ and SiLK dielectrics. He has over 100 published papers and is the holder of nine U.S. patents.

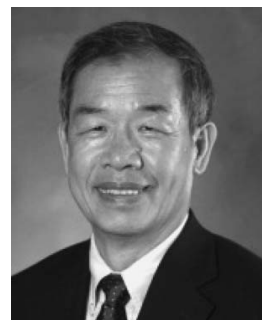

Paul S. Ho (M'91-SM'93-F'02) received the Ph.D degree in physics from Rensselaer Polytechnic Institute, Troy, NY.

He joined the Department of Materials Science and Engineering, Cornell University, Ithaca, NY, in 1966 and became an Associate Professor in 1972 In 1972, he joined the IBM T. J. Watson Research Center and became the Senior Manager of the Interface Science Department in 1985. In 1991, he joined the faculty at The University of Texas, Austin, and was appointed as Cockrell Family Regents Chair in Materials Science and Engineering, where he is currently the Director of the Laboratory for Interconnect and Packaging. His current research is in the areas of materials and processing science for interconnect and packaging applications.

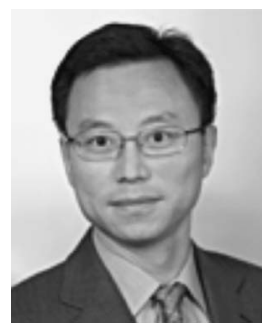

Rui Huang received the B.S. degree in theoretical and applied mechanics from the University of Science and Technology of China, Hefei, China, in 1994 and the Ph.D. degree in civil and environmental engineering, with specialty in mechanics, materials, and structures, from Princeton University, Princeton, $\mathrm{NJ}$, in 2001

In 2002, he joined the faculty at The University of Texas, Austin, where he is currently an Associate Professor of aerospace engineering and engineering mechanics and holds the position of Mrs. Pearlie Dashiell Henderson Centennial Fellowship in Engineering. His research interests include mechanics of integrated materials and structures at micro- and nanoscales, reliability of advanced interconnects and packaging for microelectronics, and mechanical instability of thin films and nanostructures. 